\title{
EL “ETHOS” DE LA CIENCIA Y EL ESTATUTO DE LA BIOÉTICA
}

\author{
Miquel Seguró ${ }^{1}$
}

Resumen: El nacimiento de la bioética no se explica sin la crítica al concepto de "ciencia" (sobre todo el positivista) que tuvo lugar durante los años anteriores a su eclosión. En este artículo nos hacemos eco de dos de sus formulaciones continentales más relevantes (K. Jaspers, M. Heidegger) para, a partir de la comprensión de su sentido, concluir que la bioética está llamada a ocupar el espacio que dicha crítica abre. Así, a consideración de una razón más plural y comprometida con los profundos motivos antropológicos y existenciales que animan las preguntas del saber, también el científico, se alza casi como un imperativo para su futuro.

Palabras clave: cientifismo, razón, finitud, existencia

\section{Science's "ethos" and the status of bioethics}

Abstract: The origins of Bioethics cannot be explained without the critique of the concept of "science" (especially the positivist) that became during the years before. In this paper we pay attention to two of its most important continental formulations (K. Jaspers, Martin Heidegger). From a general understanding of its meaning, it could be concluded that bioethics is called to occupy the space that this criticism opens. Therefore a consideration of a more pluralistic reason and committed with the anthropological and existential aims that animate the questions of knowledge, also a scientist, stands as an imperative for the future.

Key words: scientism, reason, finitude, existence

\section{O “ethos" da ciência e o estatuto da bioética}

Resumo: O nascimento da bioética não se explica sem a crítica ao conceito de "ciência" (sobretudo o positivista) que teve lugar durante os anos anteriores à sua eclosão. Neste artigo fazemos eco de duas de suas formulaçóes continentais mais relevantes (K. Jaspers, M. Heidegger) para, a partir da compreensão de seu sentido, concluir que a bioética está chamada a ocupar o espaço que a dita crítica abre. Assim, pela consideração de uma razão mais plural e comprometida com os profundos motivos antropológicos e existenciais que animam as perguntas do saber, também o científico, se eleva quase como um imperativo para seu futuro.

Palavras-chave: cientifismo, razão, finitude, existência

\footnotetext{
${ }^{1}$ Universitat Autònoma de Barcelona, España

Correspondencia: miquel.seguro@eug.es
} 
Desde que en los años ' 70 se pusiera en circulación el vocablo "bioética", el estatuto mismo de esta disciplina constituye una problemática fundamental de la bioética misma. Es más, seguramente sea la más importante. Mucho se ha debatido en torno a su posibilidad y su realidad, y en el panorama español se han destilado tradicionalmente al menos, dos posiciones al respecto(1:20): la bioética como una ética aplicada derivada de una ética cívica fundacional - A. Cortina-(2:13); la bioética como una reflexión global, también teórica, y como precisamente la ética cívica de las sociedades posindustriales -D. Gracia-(3:7). Sin embargo, ambas posiciones no explican sobre la base de una eventual oposición real, sino que responden al hecho de poner el acento en uno u otro sentido En efecto, sea porque se subraya la dependencia de una ulterior fundamentación o porque se descubre en ella las posibilidades de cartografiar los problemas fundamentales del vivir y el morir con todas sus implicaciones.

El presente artículo nace de este convencimiento. La bioética no es un apéndice de una acción ética previa, sino que es en sí misma una disquisición real de lo que involucra nuestra existencia (tanto teórica como práctica). A partir de ahí, el campo de acción de la bioética puede ser tan vasto como se quiera. Lo que en estas páginas buscamos es vislumbrar un aspecto concreto que guarda relación directa con el nacimiento de la bioética: el sentido de la crisis del concepto - sobre todo positivista- de "ciencia" como valor en sí mismo, motor sin lugar a duda del nacimiento de la bioética(4:25), y las implicaciones que de ello se derivan para la bioética presente y futura. Para ello nos centraremos en tres momentos clave de la deconstrucción mitológica de la ciencia de la primera mitad del siglo XX: la paradoja inherente a la ciencia moderna (K. Jaspers) y el desenmascaramiento de la reducción "lógica" de la ciencia (Heidegger), dos de los elementos más fundamentales del credo cientificista.

\section{La paradoja de la “ciencia moderna” según Karl Jaspers}

El curso intelectual de Karl Jaspers (1883-1969) constituye un claro y vivo ejemplo de la crisis de la ciencia positivista. Su sólida formación se afianzó en los prototipos cientificistas que por enton- ces dominaban el panorama académico en Europa. Tras iniciar los estudios de Derecho, se inclinó por la medicina, graduándose en dicha materia en 1909. Poco después comenzó a trabajar en el hospital psiquiátrico de Heidelberg y conoció de primera mano el modelo que por entonces la "verdadera" ciencia debía procurar. Jaspers se mostró crítico con la forma en que la comunidad médica de la época abordaba el tema del estudio de las enfermedades mentales, y se propuso mejorar este aspecto subrayando la incidencia que el perfil biográfico desempeña en el desarrollo de los "males del alma". Es decir, le ponía nombre y apellidos a la "enfermedad". Poco a poco, la creciente sensibilidad humanista con la que teñía su acción académica y profesional hizo que virara su interés al campo de las ciencias humanas, concretamente al de la filosofía, hecho que comportó que dejara de lado el estudio de la psicología clínica y dirigiera sus esfuerzos a la clarificación de cuestiones que atañen a los fundamentos antropológicos y existenciales del ser humano. Su conocida obra Philosophie (1932, en tres volúmenes) da cuenta de ello.

Dentro de la extensa obra de Karl Jaspers encontramos un pequeño libro (de 1931 y por lo tanto justo anterior a su obra magna) titulado $E l$ ambiente espiritual de nuestro tiempo. En él lleva a cabo un análisis de las ideas que rigen la contemporaneidad, y aunque casi haya pasado casi un siglo desde entonces - y en estos tiempos de desbocada velocidad eso es mucho-, hay en sus páginas algunos elementos que nos son todavía contemporáneos, entre los cuales la prevalencia de la visión "cuasimesiánica" de la ciencia.

Entiende Jaspers que la aparición del hombre occidental se debe, entre otra cosas, al establecimiento ya en Grecia de una racionalidad con pretensiones de validez universal, cuya finalidad es poder reducir la existencia al cálculo (ratio significa en latín justamente "cálculo"), para así poder prever su desarrollo a partir del descubrimiento de las leyes que la rigen. Con ello se pone en marcha un proceso de desdivinización del mundo, de lectura naturalista de sus sucesos, que, tras el largo paréntesis medieval, se reemprendió con el Renacimiento y, sobre todo, con el auge del desarrollo del mundo técnico y su ideal puesto en marcha a partir la Modernidad. 
Jaspers subraya que el punto clave de este desarrollo lo encontramos en la idea misma de razón. Con el progresivo avance de la racionalización se hacen cada vez más impersonales las decisiones y los procesos de su toma decisiones. Ya no son los intereses y motivos personales puntos de apoyo suficientes para validar una determinada postura o acción en el mundo, sino que ello debe dirigirse a una noción compartida y compartible (como por ejemplo lo "bueno", lo "útil", lo "necesario"), y justificar esa acción que se quiere hacer con base en ello. Hay pues un auténtico proceso de cálculo. Como consecuencia, la acción en el mundo responde a un modelo de actuación predeterminado, cuyos resultados pueden ser entonces previsibles. Junto a la racionalización, o mejor, como corolario de ella, aparece la mecanización de la acción en el mundo. La vida personal se ajusta y se amolda, como de si de un proceso febril se tratara, al discurrir predeterminado de lo que "debe" ser.

Una de las consecuencias directas de este proceso de racionalización es la despersonalización de los individuos. En un nivel social eso se traduce en la propagación de una noción unificada y homogeneizada de los individuos, que pasan a ser representantes de una misma especie, de una misma "esencia" humana y que en su totalidad representan la "masa" social. "La masa como la totalidad de los seres humanos articulados en el aparato del régimen existencial (...) es la fuerza de nuestro mundo realizándose continuamente" (5:35). Posteriormente veremos como para Horkheimer este dato constituye una señal inequívoca de la reducción de lo múltiple a lo uno, tan esencial al desarrollo de las tiranías conceptuales (y también políticas).

"El ser humano es reducido a lo general", concluye Jaspers, algo que se concreta como la reducción del individuo "a vitalidad como cosa física capaz de producción” (5:45). Eso significa que la visión de lo humano se proyecta a partir de elementos cuantitativos y de parámetros prácticamente numéricos, de ahí que sea una consecuencia lógica que la existencia humana se reduzca a la materia. El materialismo responde así a la voluntad de dominio de la existencia, ya que al reducir su efectividad a lo que materialmente se da (es decir, a lo que es palpable, cuantificable y mesurable), se la pone a disposición de la ratio humana.
Cuando Jaspers escribe su libro (1931) está convencido de la existencia de suficientes elementos que le permiten detectar una crisis de las ciencias, relacionada con la conciencia de su sentido(5:133). El estudio de las ciencias se asume por lo general como algo que ofrece prestigio, que aporta valor a quien se dedica a ello y que valida en última instancia que algo sea o no "válido". El paradigma científico, o mejor dicho, cientificista, se sitúa en el contexto de la técnica como el juez verdadero de cualquier teoría epistemológica y axiológica. Y esto para Jaspers revela una grave crisis de fondo: la crisis de la ciencia es la crisis del hombre. Es el hombre mismo quien se entrega al saber científico, y haciéndolo de manera unilateral se hace culpable de una actitud inauténtica en relación al saber y, por ende, con su propia vida. El asombro ante los resultado de "la" ciencia hace crecer en el hombre moderno su fe en ella, cada vez más ciega y por eso mismo más supersticio$\mathrm{sa}(5: 137)$.

La desnaturalización de lo que es la ciencia y, con ello, de lo que es el hombre como existencia, "como inquietud de su existencia temporal en todo momento inconclusa" (5:175), y como depositario personal de potencias creativas presentes en cada uno de nosotros de manera incomunicable pero compartible, tiene para Jaspers consecuencias importantes. Y no solamente de modo teórico o filosófico, podríamos decir, ya que estas se vislumbran día a día y llegan incluso a afectar la cotidianidad de las ciencias aplicadas (sobre todo, en el ámbito de la salud)(6).

Dos décadas más tarde, en la aparición del libro, en agosto de 1956, Jaspers pronunció una conferencia sobre la bomba atómica y el futuro de la humanidad que guarda relación con la imposibilidad de establecer una ecuación necesaria entre "ciencia" y "progreso humano". La situación de real peligro a la que la totalidad de los seres humanos estaba siendo dirigida le obligó por entonces a alertar sobre la necesidad de tomar conciencia política y ética de lo que significaba avanzar hacia un conflicto nuclear. Eso exigía sin duda ser consciente, por un lado, de que la vieja política de la astucia y la gestión del peligro y del miedo era insuficiente, porque en vez de suponer un freno al proceso de escalada de violencia, lo que comportaba era precisamente premiar la capacidad 
de mantener la tensión en el "enemigo". Y lo que precisamente se necesitaba, a ojos de Jaspers, era el establecimiento de un control mutuo a la baja, es decir, que no condujera a los contrincantes a mantenerse en una "lucha de gallos" constante. Pero, por encima de cualquiera consideración, lo que la crisis implicaba era la necesidad de reconsiderar los a priori axiológicos de la acción científica. Tomemos el caso de Albert Einstein para ilustrarlo.

Einstein fue nombrado por la prestigiosa revista Time la persona más relevante del siglo XX. No es de extrañar: su "teoría de la relatividad general" llevó a cabo una reformulación radical y completa del concepto newtoniano de gravedad y dio pie al nacimiento de la actual cosmología. Sus explicaciones sobre el efecto fotoeléctrico y sus numerosas contribuciones a la física teórica lo llevaron en 1921 a obtener el Premio Nobel de Física, aunque curiosamente no por la teoría de la relatividad como tal. Con el ascenso del nazismo, en 1932, el científico tuvo que dejar su natal Alemania y Estados Unidos no tuvo ningún reparo en acogerlo, y puso a su disposición el Instituto de Estudios Avanzados de Princeton. Nacionalizado estadounidense en 1940, murió en este municipio en 1955.

Albert Einstein fue sin lugar a dudas un pacifista convencido. Su compromiso contra la injusticia parece estar fuera de toda duda. Los acontecimientos de la Primera Guerra Mundial empujaron al joven científico a comprometerse políticamente y a involucrarse en el Partido Democrático Alemán (DDP). En 1939, ya exiliado y con el nazismo a punto de iniciar las hostilidades de la Segunda Guerra Mundial, se produce su más importante participación en cuestiones políticas. Debido al auge de la temperatura bélica, y con el riesgo real de una guerra a gran escala, algunos físicos trataron de involucrar a la Marina y al Ejército norteamericano en el proyecto atómico. La tentativa no tuvo éxito y quedó relegada. Pero una célebre carta de Albert Einstein a Roosevelt, escrita el 2 de agosto de ese mismo año, cambió el curso de los acontecimiento. En la carta, hoy de acceso público, Einstein escribió:
"En el curso de los últimos cuatro meses se ha hecho probable —a través del trabajo de Loiot en Francia así como también de Fermi y Szilard en Estados Unidos- que podría ser posible iniciar una reacción nuclear en cadena en una gran masa de uranio, por medio de la cual se generarían enormes cantidades de potencia y grandes cantidades de nuevos elementos parecidos al uranio. Ahora parece casi seguro que esto podría ser logrado en el futuro inmediato. Este nuevo fenómeno podría ser utilizado para la construcción de bombas, y es concebible - pienso que inevitable- que pueden ser construidas bombas de un nuevo tipo extremadamente poderosas. Una sola bomba de ese tipo, llevada por un barco y explotada en un puerto, podría muy bien destruir el puerto por completo, conjuntamente con el territorio que lo rodea".

Es decir, más allá de que Einstein sintiera desprecio por la violencia y las guerras, hay suficientes elementos para poder considerarlo como uno de los "padres" directos de la bomba atómica. Y es que, además de este vivo interés por informar al presidente Roosevelt de las nuevas posibilidades energéticas derivadas de la fisión en cadena de grandes masas de uranio y sus aplicaciones en el campo armamentístico, que finalmente llevaría al desarrollo de la bomba atómica, o bomba, apoyó la iniciativa de Robert Oppenheimer de comenzar el programa de desarrollo de armas nucleares conocido como "Proyecto Manhattan".

Lo sucedido después es conocido. Derrotado Hitler, la primera explosión nuclear de la historia se produjo el 16 de julio de 1945 en el desierto norte de Alamogordo, Nuevo México. Tras el "éxito" de Hiroshima y Nagasaki, las potencias "aliadas" se lanzaron a la carrera atómica porque el poder coercitivo de aquella arma era casi infinito. Ante esta situación, un año antes que Jaspers pronunciara su conferencia, Einstein, pacifista convencido y seguramente arrepentido, impulsó en julio de 1955 el conocido Manifiesto Russell-Einstein (firmado por Bertrand Russell y el propio Einstein) en el que se hacía un llamamiento a los científicos para unirse en favor de la desaparición de las armas nucleares. Este documento sirvió de inspiración para la posterior fundación de las Conferencias Pugwash, que tienen por fin implicar a los científicos en la defensa del medio ambiente, 
el desarme nuclear y la sostenibilidad económica, las cuales, en 1995, se hicieron acreedoras del Premio Nobel de la Paz.

Este caso, por paradigmático, es para Jaspers un claro indicador que el comportamiento de los investigadores comporta perplejidad. Y lo es no porque haya en Einstein un proceso dialéctico por el cual lo que en un primer momento se aceptara como óptimo después fue motivo de arrepentimiento, sino porque abre la puerta a sospechar que hay científicos que se ponen a disposición de los Estados que les sufragan sus costos de investigación a cambio de seguir disfrutando de tales ayudas (como algunos de sus colegas sí hicieron). La mayor parte de estos profesionales de la técnica quedan fascinados ante la posibilidad de poder llevar su proyecto de investigación, apunta Jaspers, dejando como "daño" colateral las posibles consecuencias de sus actos. Por ello afirma, y seguramente no falto de razones, que estos hombres tan inteligentes quieren y no quieren; se comportan como niños y luego hablan de trage$\operatorname{dias}(7: 21)$.

Una de las repercusiones del mesianismo del "bienestar científico" es que el hombre no piensa más que en su presente, en lo inmediato, y enfoca la relación costo-beneficio desde el corto plazo. Por eso Jaspers apela a una transformación, primero personal y luego colectiva, que además de asumir la paciencia dentro de los procesos de investigación, se corresponsabilice con el futuro de las generaciones venideras (Hans Jonas). La bomba atómica muestra cómo un planteamiento parco en horizontes implica la posibilidad de plantearse si una acción destructiva del hombre en relación a toda la humanidad puede ser considerada como "mala”. "¿Puede darse de nuevo el sentido de la decisión de Einstein, al aconsejar la producción de la bomba atómica ante la amenaza que suponía para el mundo el totalitarismo hitleriano?" (7:31).

Desde los meros medios técnicos no existe motivo para confiar en la fuerza persuasiva de sus argumentos, concluye. Entre otras cosas, porque no tiene esta fuerza. Los argumentos técnicos están en última instancia al servicio del hombre y su "voluntad", por lo tanto no es posible trazar una línea de continuidad entre desarrollo técni- co, bienestar y salvaguarda de la especie. El vínculo entre desarrollo técnico y valor técnico hay buscarlo más allá de la mera técnica, en lo que justamente la hace posible: la forma con la que hombre se relaciona con el mundo.

\section{Desenmascarando la esencia de la ciencia (Martin Heidegger)}

Amigo y colega de Karl Jaspers, durante las primeras décadas del siglo XX, fue Martin Heidegger (1889-1976), uno de los referentes de la filosofía de la pasada centuria. Autor de Ser y Tiempo (1927), obra capital para comprender los destinos de la filosofía actual, tiene en su haber no pocos escritos relativos a cuestiones concretas que, no por rehuir el afán sistemático de su obra magna son "obra menor". Todo lo contrario. De ellos al menos dos guardan una directa relación con la cuestión de la técnica y la ciencia, con claras aseveraciones que señalan como falaz e inauténtico el "narcisismo cientificista".

En 1953 pronunció Martin Heidegger en la $B a-$ yerische Akademie der Schönen Künste (Academia Bavaresa de Bellas Artes), una conferencia que llevaba por título "La pregunta por la técnica". En ella el filósofo alemán desarrolla la noción griega de producción (poeisis), afirmando que su semántica hay que buscarla en su deseo de traer o hacer aparecer algo desde sí mismo, desde su "pro-ducción". Así, la técnica es una manera de relacionarse con la existencia a partir del deseo de desentramar el fondo de la existencia y de desvelar (aletheia) la verdad que en ella se esconde.

Para Heidegger, el desarrollo de la técnica moderna responde en esencia al mismo proceso que rige la filosofía y técnica griegas. Es decir, también la técnica moderna responde a la voluntad de determinar una visión del mundo y la relación a establecer entre el hombre y lo que lo circunda. Técnica y ciencia moderna no son un ejercicio puro de lógica y desarrollo neutro, sino que en esencia responden a una visión del mundo y, por lo tanto, a una voluntad humana respecto a ello.

¿Y cuál es en esencia ese movimiento? Sin tratar de demonizarla, lo que Heidegger pretende es hacer al hombre consciente de los motivos que tras ella se esconden, que son, sustancialmente, dos: 
el dominio de lo natural a partir de la explicación causal de lo que se da y la unilateralidad de los postulados que la conforman. Y, latiendo tras ellos, una primera y fundamental verdad: es el sujeto humano el rector de este movimiento, por lo tanto es su interés y su intención (de dominio) lo que determinan su acción técnica(8:169). Así, en su actuar el sujeto hace del objeto de conocimiento algo que queda a su disposición.

En efecto, en alemán "objeto" corresponde a Gegenstand, pero Heidegger apunta que con la técnica el sujeto sitúa lo que lo circunda como un estar ante un "Bestand", vocablo de difícil traducción pero que semánticamente responde al sentido de "solicitud" y "disponibilidad"(9:17). Es decir, se da una relación intencional de dominio con el mundo. La técnica moderna no es otra cosa entonces que la consumación de la voluntad ancestral del hombre de tener "a la mano" la existencia, de reducirla a sus postulados y de dominar su devenir. Es más, la técnica moderna supone un episodio más de la voluntad de dominio de la existencia y de la no aceptación de su fugacidad.

En este contexto, es interesante traer a colación uno de los conceptos capitales con los que opera en su obra y que atañe a una lectura global de la historia del pensamiento: la constitución onto(teo)lógica de la metafísica. Este vocablo se refiere a un marco global, que supone que el estudio de la realidad en su concreción dada (un árbol, una casa, un libro) lleva al desarrollo de una generalidad conceptual, en la que se incluyen todas las realidades que permite desarrollar un estudio general sobre la estructura: el "ente" (el árbol, la casa y el libro son en ellos mismos entes y ejemplificadores de lo "ente"). A partir de esta noción genérica se puede desarrollar un estudio genérico de "la" realidad, pues permite reducir su complejidad a una unidad conceptual que permite y hace dúctil su estudio.

Pero hay más. Dado que en dicha "programática" debe incluirse también el sentido más alto del ente, el "máximo" ente, la onto-logía, que es el estudio de la realidad como tal, queda irremediablemente asociada a la teo-logía, que es el estudio del ente divino, o máximo ente, y así la interpretación especulativa del ente común deviene en última instancia onto-teo-logía(10:141). De este modo, apunta Heidegger, la filosofía ya no trabaja la cuestión del ente en cuanto a su entidad general (mundana, podríamos decir), sino sobre todo en cuanto a la relación que le corresponde máximamente tal entidad, el ente "supremo".

Pero lo más significativo es que esta trasposición no es inocente. Con ella el pensamiento de la realidad queda de algún modo falsificado, entiende Heidegger, porque el hombre no piensa el ser del mundo directamente, sino que lo hace en apoyo de una entidad adyacente que lo explica. ¿Por qué? Porque lo que el hombre pretende es de algún modo domar la existencia y hacer que responda a sus inquietudes y estructuras fundamentales básicas. Y puesto que la existencia es finita y relativa, y apunta sin cesar a la muerte como su elemento decisivo, el hombre busca apoyos extra existenciales para hacer soportable su discurrir. Lo auténtico para Heidegger es mirar el ser del mundo "cara a cara" y aceptar que es, en esencia, finita e incontrolable.

Pues bien, lo que antaño fue la idea del "ente máximo" (es decir, el Dios de la filosofía), en la historia de la onte(teo)logía y su falsa concepción de la realidad lo constituye la contemporaneidad la técnica. Con la técnica el ser humano se sustrae al pensamiento de la realidad en su "crudeza", de encararla en su relatividad y su escurridiza presencia, y se entrega a la ilusión de poder apresarla y ponerla a disposición de su voluntad de dominio humana. Es en sí misma un episodio más de la historia del "olvido del ser" real. Su divinización no responde a otra cosa pues que a lo in-auténtico de los propósitos del hombre en su relación con la existencia y a las armas que para ello pone en danza. El saber no es pues un factum neutro que aparece desinteresadamente en el hombre, sino que responde a una profunda intencionalidad existencial de dominio. Y eso vale para todo tipo de saber.

Es también de 1953 otro breve escrito de Heidegger, Ciencia y meditación, un texto en el que nuestro autor insiste en describirla como la manera que tenemos de afrontar el estudio de la realidad. La ciencia es una concepción del mundo, una teoría explicativa de la realidad y su andamiaje, por eso es reconocible en la visión moderna de la realidad, en las que lo científico cobra especial 
relevancia. Eso se deja ver de forma muy especial, subraya Heidegger, en el campo de la "acción". El resultado de un proceso (Ergebnis, en alemán), dice, es lo que se da entre el desarrollo de una acción y otra y por lo que "sigue", como resultado, a la consumación exitosa de su desarrollo (Erfolg) (11:44). Por lo tanto, lo que la ciencia hace es mostrar la realidad por medio de sus actuaciones, determinándola como una acción propia de su acción de conocimiento. En otras palabras, lo que la ciencia realiza es una representación de la realidad desde el prisma de su acción, que no es otra cosa que el cálculo, la relación causal y la relación de sometimiento de los "objetos" de conocimiento producidos a sus categorías.

Así se explica, por último, la necesidad de la existencia de "un" método científico. Apunta Heidegger que la creación de una "teoría" de la ciencia moderna es un proceso que asegura para cada parcela de la realidad su propio ámbito, participando todos los saberes regionales resultantes (física, química, medicina...) de una misma estructura y voluntad de conocimiento. De esta manera, cada nuevo fenómeno que aparece dentro de una rama del saber científico es elaborado y reelaborado hasta que se "adapta" al conjunto normativo y objetivo de la teoría existente, porque es en definitiva el carácter "objetivo" (y por lo tanto, mesurable y cuantificable) el espacio incuestionable y primero al cual son (re)dirigidos todos los fenómenos. Y así aparece de nuevo la verdad de la ciencia, que unificando bajo las categorías de cuantificación todo fenómeno existencial se manifiesta en su esencia: la reducción de cualquier aparecer a "objetividad", a lo seguro y apresable, a lo calculable; en definitiva, a la ratio ("cálculo", en latín) de la epistemología de los antiguos.

¿Y qué implicaciones tiene esto para el desarrollo de la ciencia en lo que atañe a sus efectos sociales, como pueda ser la medicina o las ciencias de la salud? ¿Qué sucede con ello que queda fuera del "sistema racional"? Si la finalidad escondida y ahora desenmascarada de dicho "sistema racional" es la subyugación de la realidad a los conceptos que ella establece como los óptimos (progreso), ¿cabe desterrar que la "cientificidad" de algo sea como tal un valor siempre positivo? ¿Cabe entonces sospechar que aquello que es definido como una "anomalía" es en defi- nitiva la huella de lo vivo, del elemento dinámico y siempre sorprendente de la existencia?

\section{La bioética como horizonte de la "filosofía primera"}

Una de las características fundamentales del método científico es que se apoya en un desarrollo racional siempre sujeto a revisión crítica (Karl Popper). Pero, a la vez que eso constituye su grandeza epistémica, puede convertirse también en su talón de Aquiles. "Scientia" significa saber (en alemán la relación es directa: de "wissen" — saberproviene "Wissenschaft" — ciencia-) y "ratio" cálculo. De ahí que el proceso científico opte por la noción de saber en tanto que cálculo cuando se apoya en la racionalidad en su búsqueda de unidad sistémica y unitaria del saber(12). Pero lo que entra en la denominación de "saber" no está delimitado de manera unívoca y no siempre ha tenido el mismo sentido. En efecto, en los siglos pretéritos se denominaba también a la filosofía, e incluso a la teología, "ciencia", algo hoy día impensable. Y es que no se había producido todavía el giro moderno empirista, que más tarde nutriría la descalificación positivista de dichas disciplinas.

Esta concreta revisión del estatuto de lo que es y lo que no es "saber" se explica ante todo por un contexto histórico necesitado de un proceso de emancipación antropológica. Pero tal proceso, afianzado en la ilusión de hallar un sistema de validez epistemológica, fue ya puesto en duda en los inicios del siglo XX. Que la autocomprensión de la razón como mero "cálculo" comportaba una noción estrecha de lo que significa "progresar" en el campo de las "ciencias", que asumía que el proceso positivista de cientificidad del conocimiento era neutral en cuanto a lo axiológico (si es que no hubo una asunción velada de la bondad incuestionable de su expansión), comenzó a explicitarse desde diversos puntos de vista filosóficos, como hemos visto. Aun así, las ciencias teóricas y prácticas seguían abocadas a un proceso de uniformización de sus métodos y sus propósitos bajo el paraguas de la "cientificidad" positivista y sus promesas.

Pero no solamente tales deseos no fueron asumidos (más allá del innegable avance tecnológico en todos los ámbitos, fruto directo de la investiga- 
ción científica, también la positivista), sino que tal esquema comportó una serie de elementos que desde la ciencia no podían juzgarse como algo nocivo. El desarrollo de las ciencias teóricas sistemáticas y unitarias, la necesidad de optimizar los recursos disponibles en el desarrollo de los saberes, la aplicación del esquema causal unívoco a los diferentes fenómenos de las ciencias prácticas, etc., redundaron en una situación de alarma que llevó al "gremio" sanitario (profesional y académico) de los Estados Unidos de América a plantearse que la ciencia, por sí sola, no asegura el progreso humano en los terrenos del saber y el devenir de la vida.

Seguramente, al día de hoy sea insuficiente incluso el paradigma americano de los '70 para afrontar los retos de repensar la ciencia a partir de sus paradojas. Ya no vale un simple elenco de principios o recetas de acción para encarar su realidad(13). El peso de los filtros utilitaristas y pragmáticos ya no es lo que fue, de modo que el contexto permite explorar otras racionalidades y pautas metódicas. Por eso, si la bioética se resuelve como un conjunto de principios que aplicar y una casuística a resolver sobre la base de argumentos y procedimientos "racionales", su recorrido se verá reconducido a un apéndice de los estudios científicos. Ese camino ya ha sido recorrido y ahora puede darse un paso más. Si la bioética hace honor a su nombre, es decir, es una reflexión sobre el ethos, el "carácter", de los fenómenos de la vida, entonces su campo de trabajo es todo lo que se sucede en la existencia en su relación con el no menos amplio campo de las ciencias de la salud.

La bioética nació de una insuficiencia epistemológica en relación a su raíz antropológica. Fue el hombre quien definió los parámetros de su saber científico a partir de una consideración valiosísima de la razón. Una valoración que la historia, sin embargo, ha resuelto como escueta en cuanto atañe a su unilateralidad, ya que ha comportado el menoscabo de otros espacios y de otros enfoques tanto o más importantes que el de la mera racionalidad (que, a la luz de lo que sostiene Heidegger, no es tan "mera"). Es tarea pues de la bioética señalar aquellos puntos paradójicos de las concepciones del saber; también el científico, a la luz de las complejidades y paradojas de la vida humana y de la realidad poliédrica de lo humano. En este mismo sentido, la bioética puede erigirse perfectamente en el ágora de debate de los temas clásicos de la "filosofía primera", no con un afán sistemático, perteneciente a otros momentos y a otras ilusiones premodernas, sino desde una consideración antropológica profunda y comprometida de la interacción de las esferas de lo humano con el devenir de la vida (la conciencia, la corporalidad, la interacción comunicativa, el dolor y su sentido, el vivir, el morir, la trascendencia...). En suma, una bioética interpretativa y hermenéutica consciente de sus posibilidades y también de sus condicionamientos. Una bioética que, por flexible, asume la pluralidad moral, la realidad perspectivista y la relatividad de los conceptos fundamentales como algo connatural a la experiencia antropológica. Una bioética, en definitiva, que hace de la dialogía axiológica su punto de partida.

Si, como dijera Kant (14), el hombre tiene la particularidad de estar impelido a plantearse una serie de cuestiones que no puede responder pero que tampoco puede dejar de planteárselas, entonces cada uno de los seres humanos es un ser fronterizo entre el mundo de la necesidad y el de la libertad, donde su autonomía se debate entre la finitud constitutiva de su ser y la ilimitación creativa y expresiva de sus anhelos. Un ser vivo dinámico abierto a la revisión cualitativa de los datos a la luz de su experiencia fundamental: ser contingente y finito en un mundo físico, psíquico y social dinámico.

El futuro de la bioética no es otro que el presente del hombre y su estar en el mundo. No es otro que el de la experiencia de ser en todas sus facetas, todos sus dilemas y todos sus enigmas. 


\section{Referencias}

1. Feito L. Panorama histórico de la bioética. Moralia 1997; 20: 467-494.

2. Cortina A. El estatuto de la ética aplicada. Hermenéutica crítica de las actividades humanas. Isegoría 1996; 13: 119-127.

3. Gracia D. The intellectual basis of bioethics in southern European countries. Bioethics 1993 Apr; 7(2-3): 97-107.

4. Von Potter R. Bioethics: Bridge to the Future. New Jersey: Prentice- Hall; 1971.

5. Jaspers K. El ambiente espiritual de nuestro tiempo. Barcelona: Labor; 1933.

6. Jaspers K. La práctica médica en la era tecnológica. Barcelona: Gedisa; 1988.

7. Jaspers K. La bomba atómica y el futuro del hombre. Madrid: Taurus; 19662.

8. Berciano M. La crítica de Heidegger a la metafísica occidental. Salamanca: UPSA; 1990.

9. Heidegger M. Die Frage nach dem Technik. Gesamtausgabe, vol. 7. Vorträge und Aufsätze. Frankfurt: Klostermann; 2000 .

10. Heidegger, M. Hegels Phänomenologie des Geistes. Gesamtausgabe, vol. 32. Frankfurt: V. Klostermann; 1988.

11. Heidegger M. Wissenschaft und Bessinung. Gesamtausgabe, vol. 7. Vorträge und Aufsätze. Frankfurt: Klostermann; 2000.

12. Geymonat L. Filosofía y filosofía de la ciencia. Barcelona: Editorial Labor; 1965.

13. García de las Heras JM. Debate en Bioética. Madrid: Biblioteca Nueva; 2012.

14. Kant I. Prólogo. Crítica de la razón pura. Madrid: Tecnos; 2002.

Recibido: 26 de febrero de 2014

Aceptado: 16 de marzo de 2014 